Return to the Manage Active Submissions page at http://spie.org/submissions/tasks.aspx and approve or disapprove this submission. Your manuscript will not be published without this approval. Please contact author_help@spie.org with any questions or concerns.

\title{
Solid switchable phantom for diffuse optical imaging
}

Antonio Pifferi, ${ }^{a}$ Alessandro Torricelli, ${ }^{a}$ Rinaldo Cubeddu, ${ }^{a, b}$ Giovanna Quarto, ${ }^{a}$ Rebecca Re, ${ }^{a}$ Sanathana Konugolu Venkata Sekar, ${ }^{a}$ Lorenzo Spinelli, ${ }^{b}$ Andrea Farina, ${ }^{b}$ Fabrizio Martelli, ${ }^{c}$ Heidrun Wabnitz ${ }^{d}$

${ }^{a}$ Dipartimento di Fisica, Politecnico di Milano, Milano, Italy

${ }^{b}$ Istituto di Fotonica e Nanotecnologie, Consiglio Nazionale delle Ricerche, Milano, Italy

${ }^{c}$ Dipartimento di Fisica e Astronomia dell'Università degli Studi di Firenze, Sesto Fiorentino, Firenze, Italy

${ }^{d}$ Physikalisch-Technische Bundesanstalt (PTB), Berlin, Germany

\begin{abstract}
We propose a simple and reliable solid phantom for mimicking localized absorption changes within a diffusive medium. The phantom is based on the Equivalence Relation stating that any realistic absorption inhomogeneity can be mimicked by a totally absorbing sphere of adequate volume. Applying this concept, we constructed a solid phantom holding a movable black inclusion to be positioned beneath the source-detector pair (perturbed case) or far from it (unperturbed case). Different absorption perturbations can be mimicked by changing the volume and the position of the black object both in transmittance and reflectance configuration. Time-resolved measurements of transmittance images and a lateral reflectance scan are presented.
\end{abstract}

Keywords: absorption, scattering, diffuse optics, time-resolved, optical clinical instrumentation, phantom

\section{INTRODUCTION}

The use of novel optical diagnostic tools in clinics demands for a solid culture of quality assessment and quantitative grading of instruments. ${ }^{1-4}$ Within the field of diffuse optics different initiatives were undertaken among laboratory clusters to define common protocols for performance assessment of instruments. Among those, we can recall the "BIP Protocol" ${ }^{2}$ for the assessment of basic instrument performances of time-domain diffuse optics instruments, the "MEDPHOT Protocol" ${ }^{4}$ for the quantification of the capability of instruments to retrieve the optical properties of a homogeneous medium, the "nEUROPt Protocol" ${ }^{3}$ dealing with the determination of localized absorption changes, as in the case of brain imaging, the initiative of a the multi-center American College of Radiology Imaging Network (ACRIN) to assess on phantoms the operator and instrument reliability in neadjuvant chemotheraphy monitoring, ${ }^{5}$ and finally — in the field of fluorescence imaging of tissues — phantoms were developed to characterize and compare imaging systems and to train surgeons. ${ }^{6}$

We propose here a solid phantom for the implementation of the "nEUROPt Protocol", which was initially applied using a liquid phantom. ${ }^{3,7,8}$ This phantom can be used to simulate any clinical problems related to a localized change in absorption properties, such as functional brain imaging, detection and characterization of breast tumors, monitoring of tumor reduction after neoadjuvant chemotherapy. Different solutions were proposed for the construction of inhomogeneous phantoms, see the review papers Refs. 9,10, and a recent special issue of Biomedical Optics Express ${ }^{1}$ adopting liquid-liquid, ${ }^{11}$ liquid-solid, ${ }^{12-14}$ and solid-solid structures, ${ }^{15-18}$ each of them with different advantages and criticalities. Our approach is based on a solid-solid structure constituted by a solid slab homogeneous structure and a movable black inclusion embedded in a rod that can be moved far (homogeneous case) or beneath (inhomogeneous case) the source-detector optode pair.

The use of black inclusions to mimic a realistic absorption perturbation for a wide range of optical properties, geometries and operating conditions was recently demonstrated with Monte Carlo simuations. ${ }^{19}$ A totally

Further author information:

A. Pifferi e-mail: antonio.pifferi@fisi.polimi.it

H. Wabnitz e-mail: heidrun.wabnitz@ptb.de

9325 - 5 V. 6 (p.1 of 6) / Color: No / Format: Letter / Date: 3/1/2015 11:22:17 PM

SPIE USE: DB Check, Prod Check, Notes: 
Return to the Manage Active Submissions page at http://spie.org/submissions/tasks.aspx and approve or disapprove this submission. Your manuscript will not be published without this approval. Please contact author_help@spie.org with any questions or concerns.

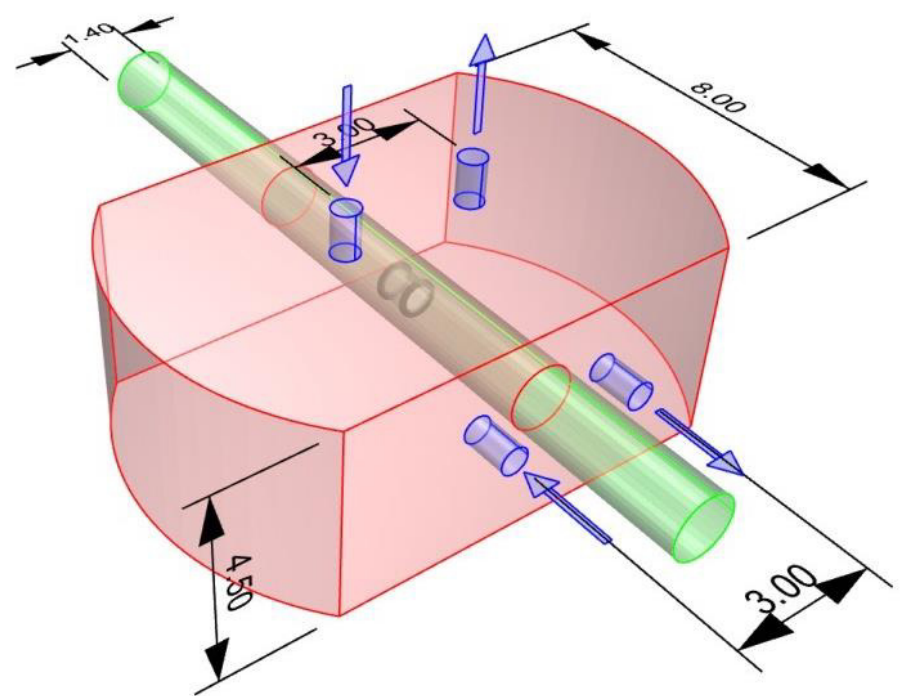

Figure 1. Scheme of the phantom. The rod with embedded black PVC object can be translated within the phantom matrix parallel to the surface. Dimensions are given in $\mathrm{cm}$.

absorbing spherical inclusion with a given volume is equivalent to an absorption inhomogeneities with different combinations of the absorption change $\Delta \mu_{a}$, volume, and shape. This equivalence was shown to be valid both for time-resolved (TR) and continuous-wave (CW) approaches and to be fairly independent of the measurement geometry, source-detector separation, and location of the inhomogeneity as well as of the background absorption coefficient $\left(\mu_{a}\right)$ apart from a few extreme cases.

\section{PHANTOM DESIGN AND CONSTRUCTION}

The scheme of the switchable solid phantom is depicted in Fig.1. It is composed of two parts: a host homogeneous phantom and a movable rod. The rod holds inside a small black PVC cylinder, and can be translated within a cylindrical hole in the host matrix set at a depth of $1.5 \mathrm{~cm}$ beneath the upper surface. The phantom matrix is made of epoxy resin, black toner, and $\mathrm{TiO}_{2}$ particles added as absorption and scattering components, respectively, following the work of Swartling et al., ${ }^{22}$ modified to better control the reproducibility and the quality of the final product. The concentration of black toner and $\mathrm{TiO}_{2}$ for the host phantom were chosen so as to yield an absorption coefficient, $\mu_{a}$, of $0.1 \mathrm{~cm}^{-1}$ and a reduced scattering coefficient, $\mu_{s}^{\prime}$, of $10 \mathrm{~cm}^{-1}$ at $690 \mathrm{~nm}$.

Four different black PVC cylinders with different dimensions (height=diameter $=3,4,5,7 \mathrm{~mm}$ ) where chosen, yielding - for a volume of $1 \mathrm{~cm}^{3}$ - four equivalent absorption perturbations $\left(\Delta \mu_{a}=0.05,0.10,0.17,0.40 \mathrm{~cm}^{-1}\right)$.

To facilitate fast and reproducible execution of the tests, the rod is connected to a stepper motor driven linear actuator that allows either manual or computer controlled (though USB driver) step-wise movements (see Figure 2). Further details can be found in Ref.23.

\section{INSTRUMENTATION FOR PHANTOM CHARACTERIZATION}

An extensive characterization of the phantom was attained using three time-domain diffuse optics systems. A time-resolved broadband near-infrared spectroscopy workstation ${ }^{24,25}$ was used to characterize the absorption and reduced scattering spectra of the bulk material in the $600 \mathrm{~nm}$ to $1200 \mathrm{~nm}$ range (data not shown). A time-domain optical mammograph ${ }^{26}$ was exploited to derive transmittance images of the phantom at different time gates. Finally, an instrument developed for functional time-resolved near-infrared spectroscopy ${ }^{27}$ was used to measure the perturbation produced by the black cylinder while passing beneath the source-detector fiber in reflectance geometry. 
Return to the Manage Active Submissions page at http://spie.org/submissions/tasks.aspx and approve or disapprove this submission. Your manuscript will not be published without this approval. Please contact author_help@spie.org with any questions or concerns.

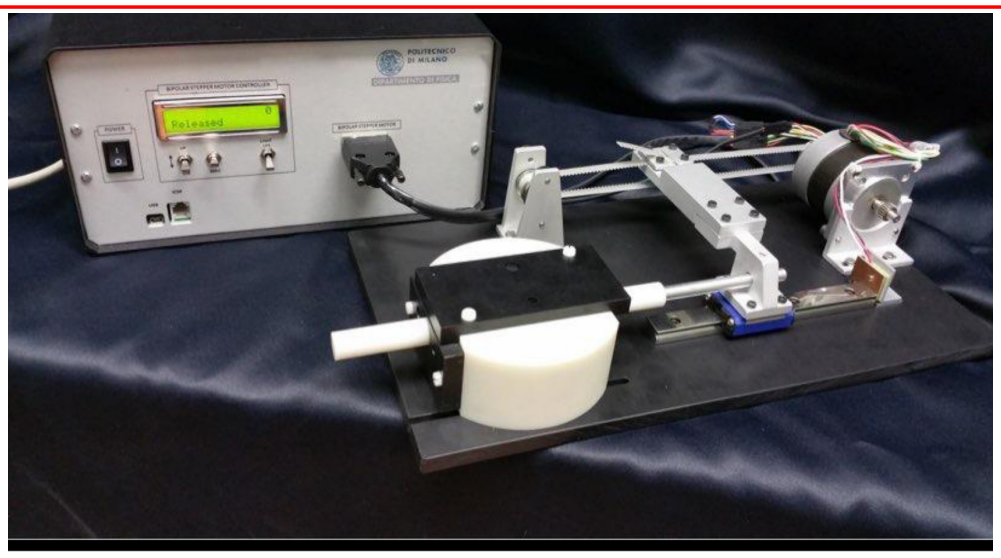

Figure 2. Photo of the switchable phantom setup. The rod is attached to a motorized linear actuator controlled by a driver permitting either manual or USB-driven translation of the rod. The upper and lateral black PVC plates facilitate light shielding and hosting of the injection-detection fibers

\section{RESULTS AND DISCUSSION}

Although in principle the rod is made of the same material as the phantom matrix, it produces a mild perturbation on time-resolved transmittance images (data not shown). This effect is possibly due to unwanted differences in optical properties or in refractive index mismatches at the interfaces. Despite the perturbation caused by the hosting rod, it is possible to extract the contribution of the black object alone by comparing the measurements with and without the black object. Figure 3 shows the contrast $C(t)=-\log \left(N(t) / N_{0}(t)\right)$ produced by the black objects, where $N(t)$ and $N_{0}(t)$ are the photon counts in the time-gated images obtained when the object is in the center of the phantom or far from it, respectively. The effect of the rod is almost eliminated, enabling a clear identification of all the four objects (rows).

The aptness of the phantom to simulate localized absorption changes in reflectance geometry was investigated using a time-domain brain imager. ${ }^{27} \mathrm{~A}$ lateral $(X$-scan) was performed by placing the fibers on the top surface of the phantom perpendicular to the rod, and translating the rod holding the black inclusion inside. Figure 4 shows the contrast $C$ for an $X$-scan of the phantom, with the inhomogeneity located at a depth of $15 \mathrm{~mm}$ and an interfiber distance of $30 \mathrm{~mm}$. The results for two different sizes of the black object are shown (left and right pane) for different time delays (see legend). As predicted by theory, the contrast depends on the size of the black object and progressively increases for higher values of the photon arrival time.

\section{CONCLUSIONS}

We have presented a new concept for the design of inhomogeneous phantoms simulating a localized absorption change based on the translation of a small black object embedded within a rod made of the same material as the phantom matrix. Different absorption perturbations can be mimicked by choosing a totally absorbing object of a proper volume. Both transmittance images and lateral reflectance scans are shown, measured using time-resolved instruments. The proposed phantom can be used either at the laboratory level, or for routine quality check of clinical instruments, or even for implementation of industrial standards.

\section{ACKNOWLEDGMENTS}

The research leading to these results has partially received funding from LASERLAB-EUROPE (grant agreement n. 284464, EC's Seventh Framework Programme) and from BABYLUX (grant agreement n. 620996, CIP-ICTPSP-2013-7). 
Return to the Manage Active Submissions page at http://spie.org/submissions/tasks.aspx and approve or disapprove this submission. Your manuscript will not be published without this approval. Please contact author_help@spie.org with any questions or concerns.

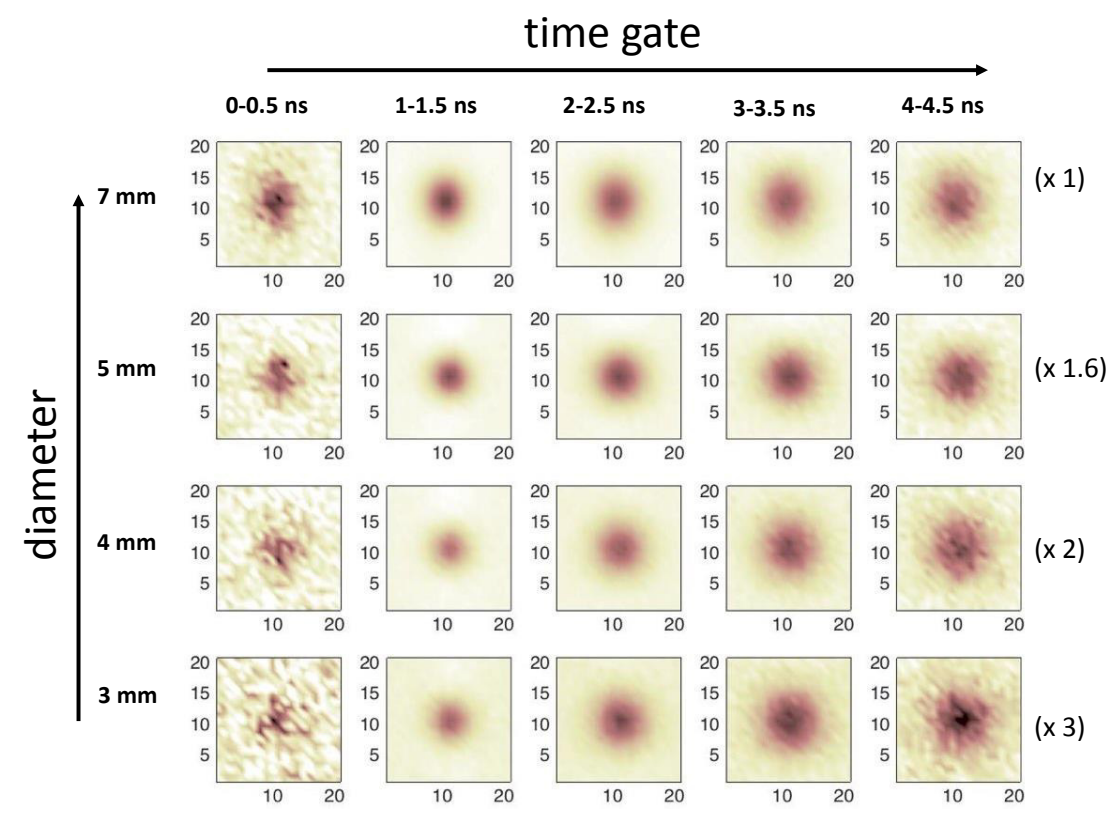

Figure 3. Transmittance images normalized to the reference state for the 4 inclusions (rows) at different time-windows (columns). The left limit of the time/window is given in the panels (ps), while their width is 500 ps. Every row is displayed with a different scale so as to adjust to the different maximum perturbation of the black object.
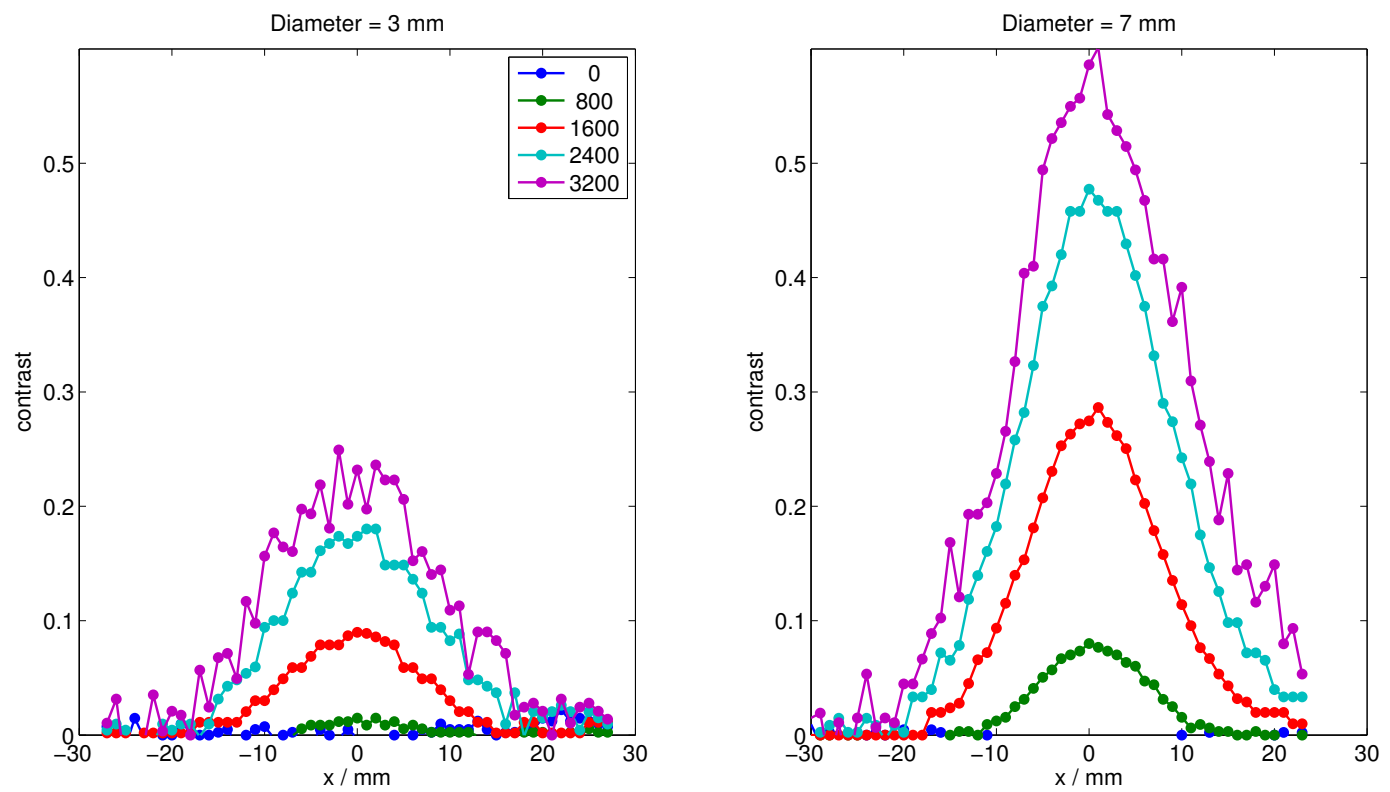

Figure 4. Contrast $C$ for reflectance measurements for two sizes of the black object (left and right pane) as a function of the lateral displacement ( $X$-Scan). Different time-delays (ps) are shown (legend). 
Return to the Manage Active Submissions page at http://spie.org/submissions/tasks.aspx and approve or disapprove this submission. Your manuscript will not be published without this approval. Please contact author_help@spie.org with any questions or concerns.

\section{REFERENCES}

[1] J. Hwang, J. C. Ramella-Roman, and R. Nordstrom, "Introduction: Feature issue on phantoms for the performance evaluation and validation of optical medical imaging devices," Biomed. Opt. Express 3, (6), 1399-1403 (2012).

[2] H. Wabnitz et al., "Performance assessment of time domain optical brain imagers, part 1: basic instrumental performance protocol," J. Biomed. Opt. 19, (8), 086010 (2014).

[3] H. Wabnitz et al., "Performance assessment of time-domain optical brain imagers, part 2: nEUROPt protocol," J. Biomed. Opt. 19, (8), 086012 (2014).

[4] A. Pifferi, A. Torricelli, A. Bassi, P. Taroni, R. Cubeddu, H. Wabnitz, D. Grosenick, M. Möller, R. Macdonald, J. Swartling, T. Svensson, S. Andersson-Engels, R. L. P. van Veen, H. J. C. M. Sterenborg, J.-M. Tualle, H. L. Nghiem, S. Avrillier, M. Whelan, and H. Stamm, "Performance assessment of photon migration instruments: the medphot protocol," Appl. Opt. 44, (11), 210414 (2005).

[5] A. E. Cerussi, R. Warren, B. Hill, D. Roblyer, A. Leproux, A. F. Durkin, T. D. O'Sullivan, S. Keene, H. Haghany, T. Quang, W. M. Mantulin, and B. J. Tromberg, "Tissue phantoms in multicenter clinical trials for diffuse optical technologies," Biomed. Opt. Express 3, (5), 966-971 (2012).

[6] A. M. De Grand, S. J. Lomnes, D. S. Lee, M. Pietrzykowski, S. Ohnishi, T. G. Morgan, A. Gogbashian, R. G. Laurence, and J. V. Frangion, "Tissue-like phantoms for near-infrared fluorescence imaging system assessment and the training of surgeons," J. Biomed. Opt. 11, (1), 014007 (2006).

[7] F. Martelli, P. Di Ninni, G. Zaccanti, D. Contini, L. Spinelli, A. Torricelli, R. Cubeddu, H. Wabnitz, M. Mazurenka, R. Macdonald, A. Sassaroli, and A. Pifferi, "Phantoms for diffuse optical imaging based on totally absorbing objects, part 2: experimental implementation," J. Biomed. Opt. 19, (7), 076011 (2014).

[8] L. Spinelli, M. Botwicz, N. Zolek, M. Kacprzak, D. Milej, P. Sawosz, A. Liebert, U. Weigel, T. Durduran, F. Foschum, A. Kienle, F. Baribeau, S. Leclair, J.-P. Bouchard, I. Noiseux, P. Gallant, O. Mermut, A. Farina, A. Pifferi, A. Torricelli, R. Cubeddu, H.-C. Ho, M. Mazurenka, H. Wabnitz, K. Klauenberg, O. Bodnar, C. Elster, M. Bénazech-Lavoué, Y. Bérubé-Lauzière, F. Lesage, D. Khoptyar, A. A. Subash, S. AnderssonEngels, P. Di Ninni, F. Martelli, and G. Zaccanti, "Determination of reference values for optical properties of liquid phantoms based on Intralipid and India ink," Biom. Opt. Express 5, (7), 2037-2053 (2014).

[9] G. Lamouche, B. F. Kennedy, K. M. Kennedy, C. E. Bisaillon, A. Curatolo, G. Campbell, V. Pazos, and D. D. Sampson, "Review of tissue simulating phantoms with controllable optical, mechanical and structural properties for use in optical coherence tomography," Biomed. Opt. Express 3, (6), 1381-1398 (2012).

[10] B. W. Pogue and M. S. Patterson, "Review of tissue simulating phantoms for optical spectroscopy, imaging and dosimetry," J. Biomed. Opt. 11, (4), 041102 (2006).

[11] H. Jiang, K. D. Paulsen, U. L. Österberg, and M. S. Patterson, "Frequency-domain optical image reconstruction in turbid media: An experimental study of single-target detectability," Appl. Opt. 36, (1), 52-63 (1997).

[12] J. P. Culver, R. Choe, M. J. Holboke, L. Zubkov, T. Durduran, A. Slemp, V. Ntziachristos, B. Chance, and A. G. Yodh, "Three-dimensional diffuse optical tomography in the parallel plane transmission geometry: Evaluation of a hybrid frequency domain/continuous wave clinical system for breast imaging," Med. Phys. 30, (2), 235-247 (2003).

[13] R. Cubeddu, A. Pifferi, P. Taroni, A. Torricelli, and G. Valentini, "Time-resolved imaging on a realistic tissue phantom: $\mu_{s}^{\prime}$ and $\mu_{a}$ images versus time-integrated image," Appl. Opt. 35, (22), 4533-4540 (1996).

[14] S. Carraresi, T. S. M. Shatir, F. Martelli, and G. Zaccanti, "Accuracy of a perturbation model to predict the effect of scattering and absorbing inhomogeneities on photon migration," Appl. Opt. 40, (25), 4622-4632 (2001).

[15] R. Cubeddu, A. Pifferi, P. Taroni, A. Torricelli, and G. Valentini, "A solid tissue phantom for photon migration studies," Phys. Med. Biol. 42, (10), 1971-1979 (1997).

[16] J. C. Hebden, T. C. J. Brunker, B. D. Price, A. P. Gibson, and N. L. Everdell, "An electrically-activated dynamic tissue-equivalent phantom for assessment of diffuse optical imaging systems," Phys. Med. Biol. 53, (2), 329-337 (2008). 
Return to the Manage Active Submissions page at http://spie.org/submissions/tasks.aspx and approve or disapprove this submission. Your manuscript will not be published without this approval. Please contact author_help@spie.org with any questions or concerns.

[17] A. Gibson, R. M. Yusof, H. Dehghani, J. Riley, N. Everdell, R. Richards, J. C. Hebden, M. Schweiger, S. R. Arridge, and D. T. Delpy, "Optical tomography of a realistic neonatal head phantom," Appl. Opt. 42, (16), 3109-3116 (2003).

[18] T. Funanen, H. Atsumori, M. Kiguchi, Y. Tanikawa, and E. Okada, "Dynamic phantom with two stagedriven absorbers for mimicking hemoglobin changes in superficial and deep tissues," Journal of Biomedical Optics 17, (4), 047001 (2012)

[19] F. Martelli, A. Pifferi, D. Contini, L. Spinelli, A. Torricelli, H. Wabnitz, R. Macdonald, A. Sassaroli, and G. Zaccanti, "Phantoms for diffuse optical imaging based on totally absorbing objects, part 1: Basic concepts," J. Biom. Opt. 18, (1), 66014 (2013).

[20] R. Barbour, R. Ansari, R. Al abdi, H. L. Graber, M. B. Levin, Y. Pei, C. H. Schmitz, and Y. Xu, "A programmable laboratory testbed in support of evaluation of functional brain activation and connectivity Validation of near infrared spectroscopic (NIRS) imaging using programmable phantoms," in Design and Performance Validation of Phantoms Used in Conjunction With Optical Measurements of Tissue Proceedings of SPIE 6870, 687002 (2008).

[21] R. Barbour, H. Graber, Y. Xu, Y. Pei, C. Schmitz, D. Pfeil, A. Tyagi, R. Andronica, D. Lee, S.-L. S. Barbour, J. Nichols, and M. Pflieger, "A programmable laboratory testbed in support of evaluation of functional brain activation and connectivity," IEEE Trans Neural Syst Rehabil Eng. 20, (2), 170-183 (2012).

[22] J. Swartling, J. S. Dam, S. Andersson-Engels, "Comparison of spatially and temporally resolved diffusereflectance measurement systems for determination of biomedical optical properties," Appl.Opt. 42, (22), 4612-4620 (2003).

[23] A. Pifferi, A. Torricelli, R. Cubeddu, G. Quarto, R. Re, S. Konugolu, L. Spinelli, A. Farina, F. Martelli, H. Wabnitz, 'Mechanically switchable solid inhomogeneous phantom for performance tests in diffuse imaging and spectroscopy," J. Biom. Opt. submitted (2015).

[24] A. Pifferi, A. Farina, A. Torricelli, G. Quarto, R. Cubeddu, P. Taroni, "Time-domain broadband near infrared spectroscopy of the female breast: a focused review from basic principles to future perspectives," J. Near Infrared Spectrosc. 20, (1), 223-235 (2012).

[25] I. Bargigia, A. Nevin, A. Farina, A. Pifferi, C. D'Andrea, M. Karlsson, P. Lundin, G. Somesfaleand, and S. Svanberg, "Diffuse optical techniques applied to wood characterisation," J. Near Infrared Spectrosc. 21, 259-?268 (2013).

[26] P. Taroni, A. Pifferi, E. Salvagnini, L. Spinelli, A. Torricelli, and R. Cubeddu, "Seven-wavelength timeresolved optical mammography extending beyond $1000 \mathrm{~nm}$ for breast collagen quantification," Opt. Express 17, 15932-15946 (2009).

[27] R. Re, D. Contini, M. Turola, L. Spinelli, L. Zucchelli, M. Caffini, R. Cubeddu, and A. Torricelli, "Multichannel medical device for time domain functional near infrared spectroscopy based on wavelength space multiplexing," Biomed. Opt. Express 4, 2231-2246 (2013). 